\title{
Kinase Activation by Small Conformational Changes
}

\author{
2 Elias D. López, ${ }^{\dagger}$ Osvaldo Burastero, ${ }^{\dagger}{ }^{\dagger}$ Juan Pablo Arcon, ${ }^{\dagger}$ Lucas A. Defelipe, ${ }^{\dagger}$ Natalie G. Ahn, ${ }^{\ddagger}$ \\ 3 Marcelo A. Marti, ${ }^{\dagger}$ and Adrian G. Turjanski* ${ }^{\dagger}$ (†) \\ $4{ }^{\dagger}$ Departamento de Química Biológica, Facultad de Ciencias Exactas y Naturales, Universidad de Buenos Aires, Ciudad Autónoma de \\ 5 Buenos Aires, Argentina \\ $6{ }^{*}$ Department of Chemistry and Biochemistry, University of Colorado, Boulder, Colorado 80309, United States
}

\section{S Supporting Information}

8 ABSTRACT: Protein kinases (PKs) are allosteric enzymes 9 that play an essential role in signal transduction by regulating a 10 variety of key cellular processes. Most PKs suffer conforma11 tional rearrangements upon phosphorylation that strongly 12 enhance the catalytic activity. Generally, it involves the 13 movement of the phosphorylated loop toward the active site 14 and the rotation of the whole C-terminal lobe. However, not all kinases undergo such a large configurational change: The 15 MAPK extracellular signal-regulated protein kinases ERK1 and ERK2 achieve a 50000 fold increase in kinase activity with only 16 a small motion of the C-terminal region. In the present work, we used a combination of molecular simulation tools to 17 characterize the conformational landscape of ERK2 in the active (phosphorylated) and inactive (unphosphorylated) states in 18 solution in agreement with NMR experiments. We show that the chemical reaction barrier is strongly dependent on ATP 19 conformation and that the "active" low-barrier configuration is subtly regulated by phosphorylation, which stabilizes a key salt 20 bridge between the conserved Lys52 and Glu69 belonging to helix-C and promotes binding of a second Mg ion. Our study 21 highlights that the on-off switch embedded in the kinase fold can be regulated by small, medium, and large conformational 22 changes.

24 Protein kinases (PKs) are allosteric enzymes that play an 25 essential role in signal transduction, regulating many biological 26 processes. PKs have evolved to be dynamic molecular switches 27 that can be controlled by dimerization, membrane recruitment, 28 and phosphorylation. A large set of serine/threonine and 29 tyrosine PKs are phosphorylated in their activation segment30 loop leading to a conformational change that strongly enhances 31 their catalytic activity, and therefore research efforts have 32 focused on understanding how kinase activity is dynamically 33 regulated by this configurational transition. In many PKs, a 34 large conformational change is observed upon activation, 35 involving the opening of the A-loop, the relative alignment of $36 \mathrm{~N}$-lobe and C-lobe, and the rotation of the $\alpha \mathrm{C}$-helix. The 37 "closure/rotation" allows important residues necessary for 38 catalysis to accommodate in the active site. ${ }^{1}$

39 Extracellular activated protein kinases ERK/MAPKs (ERK1 40 and ERK2) are the most extensively studied members of the 41 mitogen-activated protein kinase (MAPK) subfamily of PKs 42 and aberrant activation of their signaling pathway is a frequent 43 event in many human malignancies. ${ }^{2,3}$ Because the ERK 44 signaling pathway is an attractive target for cancer chemo45 therapy, interest in its components has exploded in the past 46 few years. ${ }^{4,5}$ MAPKs are characterized by their requirement of 47 dual phosphorylation at conserved threonine (Thr) and 48 tyrosine (Tyr) residues for activation ${ }^{6,7}$ and their specific 49 activity toward Ser/Thr residues of the substrate proteins/ 50 peptides, which are always followed by a proline residue. ${ }^{8}$
Structural and biochemical studies have extensively charac- 51 terized the activation process and the catalytic activity of 52 MAPKs; and there is compelling evidence that MAPKs 53 facilitate substrate recognition through docking interactions 54 outside the active site, where ATP binding and phosphate 55 transfer occur. 9

Conformational changes accompanying the activation of 57 ERK2, albeit small, have been documented by X-ray structures 58 of the inactive, unphosphorylated (OP-ERK2) ${ }^{10}$ and active, 59 dual-phosphorylated (2P-ERK2) forms. ${ }^{6}$ Phosphorylation 60 rearranges the activation loop, leading to new ion-pair 61 interactions between phospho-Thr and phospho-Tyr residues 62 with the basic residues in the $\mathrm{N}$ - and $\mathrm{C}$-terminal lobes of the 63 kinase core structure. Other structural changes upon activation 64 include the folding of a $3_{10}$ helix at loop L16 which exposes a 65 hydrophobic zipper that forms the domain interface in the 66 dimer, ${ }^{11}$ and a small domain rotation $\left(5^{\circ} \text { over the C-lobe }\right)^{6}{ }_{67}$ (Figure 1A,B) 1. Recent studies show the importance of the $68 \mathrm{fl}$ loop L16 for the stabilization of $\alpha \mathrm{C}$-helix and also provide a 69 structural framework for allosteric coupling between peptide 70 binding at the $\mathrm{D}$-domain docking groove and catalytic control 71 via $\alpha$-helix positioning and interlobe motion in ERK2. ${ }^{12,13} 72$ More recently, Carr-Purcell-Meiboom-Gill (CPMG) relax- 73

Special Issue: Molecular Simulation in Latin America: Coming of Age

Received: September 13, 2019

Published: November 12, 2019 

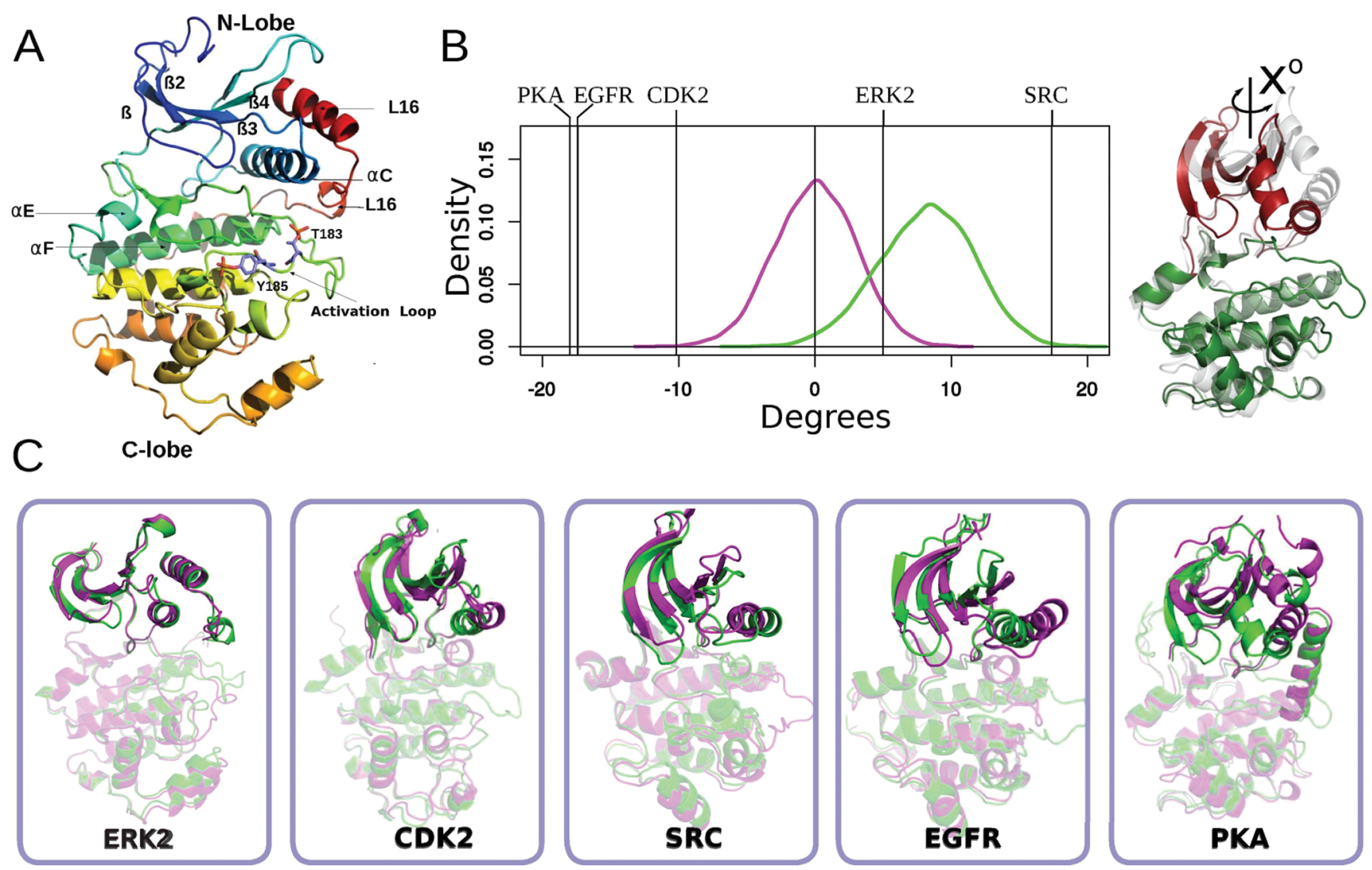

Figure 1. Activation promotes conformational changes in helix-C and the $\mathrm{N}$-terminal lobe. (A) Cartoon representation of the structure of active ERK2. Color changes from blue (N-terminal) to red (C-terminal). Secondary structures elements are labeled accordingly. (B) Kernel density estimation of the N-lobe to C-lobe rotational angle of the ERK2 taken from $1 \mu$ s long MD simulations (inactive shown in magenta, active in green). Vertical lines correspond to the change in the angle observed for the crystallographic structures of different kinases, the sign represents the direction of rotation of the active conformer respect to the inactive form. (C) Inactive (magenta) and active (green) conformations of five different kinases are shown (after C-lobe alignment) in order to illustrate the structural changes of the C-helix and N-terminal lobes.

74 ation dispersion experiments, an NMR-based method to 75 monitor protein dynamics on slow time scales (100-2000 $76 \mathrm{~s}$ ), suggested that ERK2 displays a dynamic equilibrium 77 between two conformational states. 0P-ERK2 presents mainly 78 an inactive conformation constrained from domain motions, 79 and phosphorylation is proposed to shift the equilibrium to 80 favor the active conformer. ${ }^{14}$ However, since 0P-ERK2 and 2P81 ERK2 structures are very similar, the structural differences 82 responsible for the increase in activity and CPMG reported 83 conformational changes, are far from clear.

84 The structural determinants that control ERK2 and the 85 chemical step if other kinases are also still poorly understood. 86 In most PKs in the active state, an invariant active site lysine 87 (Lys52 in ERK2) residue plays a critical role in stabilizing the 88 transition-state, by coordination to the $\alpha$ and $\beta$ phosphoryl 89 group oxygen atoms of ATP. ${ }^{15,16}$ Substitution of this residue to 90 arginine or alanine in ERK2 results in a dramatic reduction in 91 the turnover rate, with little effect on ground state ATP 92 binding. ${ }^{7,17}$ Similarly, in inactive 0P-ERK2, Lys52 does not 93 make contact with ATP (PDB ID 4GT3), explaining its 94 reduced phosphotransfer kinetics, despite little difference in 95 ATP affinity. ${ }^{18,19}$ Dual phosphorylation is presumed to 96 optimize the alignment of Lys52, activating the protein, as 97 supported by a set of inhibitors that despite allowing the DFG 98 motif to adopt an active conformation disrupt the key salt 99 bridge between the catalytic Lys52 and a conserved glutamic 100 acid $^{20}$ (Glu69 in ERK2).
Finally, from a chemical mechanism viewpoint, there are 101 several recent theoretical studies on kinases mainly based on 102 hybrid quantum-mechanics/molecular-mechanics (QM/MM) 103 methods. ${ }^{21-24}$ Earlier studies of CDK2, for example, suggested 104 that an Asp residue serves as the general base to activate the 105 Ser nucleophile. The corresponding transition state features a 106 dissociative metaphosphate-like structure, stabilized by the $\mathrm{Mg} 107$ ion and several hydrogen bonds. ${ }^{21}$ Specifically concerning 108 MAPKs, previous studies from our group using an energy 109 minimization scheme, showed for ERK2 a moderate barrier 110 $(17 \mathrm{kcal} / \mathrm{mol})$ for the active state, and the key role played by 111 Lys52 to strongly stabilize the transition state. ${ }^{17}$

112

A summary of the previous studies on ERK2 leads to the 113 following conclusions: (i) the structures of ERK2 in the crystal 114 active and inactive states are very similar in terms of catalytic 115 pocket, (ii) ATP binding affinity is not significantly affected, 116 (iii) solution NMR and H/D experiments suggest the presence 117 of two conformations in OP-ERK2 that shift to one 118 configuration upon phosphorylation. None of these provides 119 a definite answer to how at the molecular level dual 120 phosphorylation results in ERK2 activation, and the role 121 played by protein conformational dynamics in catalysis. In the 122 present work, we have used multiscale molecular simulation 123 tools, such as quantum mechanics (QM) and molecular 124 mechanics (MM) simulations, to unravel the coupling between 125 conformational dynamics and catalysis in ERK2. Our results 126 provide evidence that the chemical reaction barrier is strongly 127 
128 dependent on ATP conformation. In turn, the active low129 barrier configuration is subtly regulated by phosphorylation 130 which allows the activation loop to reorganize a key salt bridge 131 between the conserved Lys52 and Glu69 in helix-C and 132 promotes the proper binding of the second $\mathrm{Mg}$ ion, resulting in 133 a suitable conformation for catalysis. This mechanism, which 134 seems to be conserved in many Ser/Thr protein kinases, 135 explains why despite a lack of significant overall differences 136 between the active and inactive ERK2 structures there is a 13750000 fold increase in catalytic activity upon phosphorylation.

\section{METHODS}

139 ERK2 Starting Structures. The starting structures of 140 nonphosphorylated and dual-phosphorylated ERK2 were 141 obtained from the Protein Data Bank (entries 1ERK, ${ }^{25}$ $1422 \mathrm{ERK}^{6}$ respectively). The catalytically competent complex 143 was built as in our previous QM/MM study of ERK2 reaction 144 mechanism. ${ }^{17}$ Briefly, we added to the dual-phosphorylated 145 ERK2 structure the ATP and two Mg ions using as a template 146 the structure of $\mathrm{p} 38 \gamma$ (PDB 1CM8). ${ }^{26}$ This structure 147 corresponds to a highly similar MAPK which was crystallized 148 phosphorylated, bound to ATP, and has two octahedrally 149 coordinated $\mathrm{Mg}$ binding sites. In other words, we incorporated 150 the ATP and two Mg ions to the 2P-ERK2 structure respecting 151 the coordination found in the crystal structure of $\mathrm{p} 38 \gamma$ 152 (1CM8). We analyzed the $\mathrm{Mg}$ ions coordination for other 153 protein kinases but we did not find significant differences 154 (Table S1). Subsequently, we added the phosphoacceptor 155 substrate as described in our previous work, with the $\mathrm{OH}$ 156 group of Thr pointing toward the $\gamma$-phosphate of ATP and the 157 conserved proline in a pocket comprised by the phospho-Thr 158 in the P+1 site. ${ }^{17}$ Last, the ATP-bound conformation for the 159 unphosphorylated form of ERK2, was built taking into account 160 the ATP and the $\mathrm{Mg}$ ion disposition from the inactive ERK2 161 structures $4 \mathrm{~S} 32$ and $4 \mathrm{GT} 3$ (in addition to 1CM8).

162 A summary of the models used in this work is shown in 163 Table 1. In all the ATP-bound structures the Mg binding Site I

Table 1. ERK2 Models

\begin{tabular}{lccc}
\multicolumn{1}{c}{ name } & ATP/2Mg & Phos-Tyr and Phos-Thr & substrate \\
OP-ERK2 & - & - & - \\
P-ERK2-ATP & $\sqrt{ }$ & - & - \\
P-ERK2 & - & - & - \\
P-ERK2-ATP & $\sqrt{ }$ & $\sqrt{ }$ & - \\
P-ERK2-ATP-substrate & $\sqrt{ }$ & $\sqrt{ }$ & $\sqrt{ }$ \\
\hline
\end{tabular}

164 (MSI) is built by bidentate coordination to Asp165, ATP-P $\beta$, 165 and two water molecules, the $\mathrm{Mg}$ binding site II (MSII) by 166 Asn 152, Asp165 ATP-P $\alpha$, and one water molecule (Figure S1). 167 The modeled structures are provided as Supporting 168 Information.

169 Classical Simulation Parameters. All classical simula170 tions were performed with the AMBER16 package of 171 programs. ${ }^{27}$ The starting structures were solvated with an 172 isometric truncated octahedron of TIP $3 \mathrm{P}^{28}$ water molecules 173 extending a minimum of $12 \AA$ beyond protein edge. Hydrogen 174 atoms were added with the tleap module of the Amber 175 Program package. ${ }^{27}$ Standard protonation states were assigned 176 to titrable residues ( $\mathrm{D}$ and $\mathrm{E}$ are negatively charged, $\mathrm{K}$ and $\mathrm{R}$ 177 positively charged) for all protocols. Histidine protonation was 178 assigned to favoring formation of hydrogen bonds in the crystal 179 structure. All standard residues were represented using the
AMBER14SB force field. ${ }^{29}$ Parameters for phospho-Tyr and 180 phospho-Thr were taken from Craft et al. ${ }^{30}$ and the ATP 181 parameters from Meagher et al. ${ }^{31}$ In all simulations the 182 particle-mesh Ewald method ${ }^{32}$ was used with a nonbonded 183 cutoff of $12 \AA$ for computing electrostatic interactions, and the 184 Langevin thermostat, Berendsen barostat to maintain the 185 desired temperature and pressure, respectively. ${ }^{33,34}$ All hydro- 186 gen bonds were kept rigid by using the SHAKE algorithm, and 187 the hydrogen mass repartitioning scheme was employed. The 188 scheme scales all hydrogen masses by a factor of 3, allowing the 189 use of a 4 fs time step for integrating Newton's equations. ${ }^{35,36} 190$ Each system was first gently heated to $300 \mathrm{~K}$ for 200 ps at 191 constant volume and using a soft harmonic restraint to all C- 192 alpha carbons $\left(1 \mathrm{kcal} / \mathrm{mol} \AA^{2}\right)$. Subsequently, 1 ns constant 193 temperature and pressure MD simulations were performed to 194 equilibrate the system density without any additional restraints. 195 Production simulations consisted of $1 \mu \mathrm{s}$ long molecular 196 dynamics (MD) simulations for the apo (OP-ERK2 and 2P- 197 ERK2) and holo (OP-ERK2-ATP and 2P-ERK2-ATP) states. 198

Domain Orientation. Domain closure/rotation was 199 calculated by aligning the $\mathrm{C}$-terminal lobe and then computing 200 the interlobe rotation ( $\mathrm{N}$ - to $\mathrm{C}$-lobe rotation). For this 201 purpose, a homemade script using pymol was used (https:// 202 pymolwiki.org/index.php/Angle_between_domains). The 203 PDB IDs used to calculate the interdomain rotation of Figure 204 1 were the following: PKA (4DFY, $\left.{ }^{37} 1 \mathrm{~J}^{3} \mathrm{H}^{38}\right), \mathrm{CDK} 2205$ $\left(4 \mathrm{KD}^{39}{ }^{39} 3 \mathrm{QHW}^{40}\right)$, EGFR (2GS6, 2GS7 $\left.{ }^{41}\right), \operatorname{Src}\left(2 \mathrm{SRC}{ }^{42} 206\right.$ $\left.3 \mathrm{DQW}^{43}\right)$ and ERK2 $\left(1 \mathrm{ERK}^{25} 2 \mathrm{ERK}^{6}\right)$. The figures were 207 generated using the PyMol molecular graphics software 208 (v.1.7.2.1; Schrödinger LLC). The sign represents the 209 direction of rotation. Negative numbers imply a clockwise 210 rotation of the active form (into the direction of the ATP 211 pocket, with the C-lobe below and the $\mathrm{N}$-lobe above) while 212 positive numbers imply a counterclockwise rotation. 213

Essential Mode (EM) Analysis. To analyze the overlap 214 between each protein state dynamics (OP-ERK2, 2P-ERK2, 0P- 215 ERK2-ATP, and 2P-ERK2-ATP) the structural transition 216 between them, we computed the "transition EM" correspond- 217 ing to the first EM derived from the covariance matrix obtained 218 from a combined trajectory, which is simply built by joining 219 the two independent simulations. This "transition EM" 220 represents a collective coordinate that describes the conforma- 221 tional transition and can be used to analyze the configurational 222 space, along this coordinate, explored by any simulation by 223 simply projecting the corresponding trajectory on the 224 corresponding EM, according to eq 1

$$
P_{N}(t)=\mathbf{V} \cdot r(t)
$$

where $\mathbf{V}$ is the transition EM vector and $r(t)$ is the protein 227 conformation at time $t$. Projections are measured in angstroms, 228 and the value corresponds to the overall deviation from the 229 mean structure along the projected transition mode. After 230 projecting the selected MD simulation along the selected 231 mode, the normalized histograms were computed. For more 232 details about EM analysis see the references. ${ }^{44}$

233

Umbrella Sampling (US). US was used to determine the 234 strength of the conserved salt bridge between Lys52 and 235 Glu69, and to estimate the free energy change associated with 236 Phe-IN and Phe-OUT states of the L16 loop, using as 237 conformational coordinate the distances between Lys52: $\epsilon-\mathrm{NH}_{3}^{+} 238$ and Glu69:C $\delta$ and between the alpha carbons of the Ile72 and 239 the Phe329, respectively. Windows were evenly spaced with a 240 $0.5 \AA$ separation distance and simulated for $2 \mathrm{~ns}$ each using a 241 
242 force constant of $30 \mathrm{kcal} / \mathrm{mol} / \AA^{2}$. It should be noted that the 243 initial conformations of the OP-ERK2-ATP and 2P-ERK2-ATP 244 for the umbrella sampling protocol, were built with a properly 245 established salt bridge. The potential of mean force was 246 calculated as implemented in WHAM. ${ }^{45}$ Histogram overlap 247 and convergence of the US simulations are respectively shown 248 in Figure S2 and Figure S3. Statistical uncertainty was 249 determined using 50 bootstrap samples and was in all cases 250 below $0.013 \mathrm{kcal} / \mathrm{mol}$ (Figure S4).

251 Thermodynamic Integration (TI). To analyze the effect 252 of dual-phosphorylation in the Mg ion affinity at the MSI site 253 we computed the binding affinity in both the OP-ERK2-ATP 254 and 2P-ERK2-ATP states using the thermodynamic cycle 255 depicted in Figure S5. The relative free energies were 256 calculated using AMBER $16^{27}$ with the AMBER 14SB force 257 field $^{29}$ using the TI implementation in pmemd. ${ }^{46}$ The starting 258 structures for the TI calculation were taken from snapshots 259 corresponding to an equilibrated MD trajectory, and the free 260 energy perturbation calculations were performed by alchemi261 cally modifying the complex for the "disappearing" of the $\mathrm{Mg}$ 262 ion at the MSI site (MgI ion). This was achieved by scaling the 263 electrostatic interactions and van der Waals interactions 264 simultaneously and using soft-core potentials. ${ }^{47} \mathrm{We}$ varied 265 the coupling parameter in 0.1 steps from 0 to 1 explicitly with a 266 total simulation time per $\lambda$ window of $1.5 \mathrm{~ns}$. To get converged $267 \mathrm{~d} v / \mathrm{d} \lambda$ values, we kept the last nanosecond of the trajectory per 268 window. $\Delta \Delta G$ of $\mathrm{Mg}$ binding was estimated as $\Delta G_{0 P}-\Delta G_{2 P}$ 269 (Table 2). Each $\Delta G$ value was estimated by averaging the

Table 2. Summary of TI Calculations

$\begin{array}{clc}\text { name } & \text { initial structure }(\lambda=0) & \text { final structure }(\lambda=1) \\ \Delta G_{0 P} & \text { OP-ERK2-ATP with MgI ion and } & \text { OP-ERK2-ATP with MgII } \\ & \text { MgII ion } & \text { ion } \\ \Delta G_{2 P} & \begin{array}{c}\text { 2P-ERK2-ATP with MgI ion and } \\ \text { MgII ion }\end{array} & \text { 2P-ERK2-ATP with MgII } \\ & \text { ion }\end{array}$

270 binding energy of 3 replicates, reaching a total of 33 windows 271 per state and a total simulation time of $50 \mathrm{~ns}$. Statistical 272 analysis and estimation of the binding free energy for each 273 replica were made with the python script of MBAR. ${ }^{48}$ The 274 performed TI calculations are shown in Table 2.

275 Determination of the Reaction Free Energy Profile 276 Using QM/MM and Multiple Steered Molecular Dynam277 ics (MSMD) Strategy. To determine the free energy profile 278 (FEP) of the phospho-transfer reaction catalyzed by ERK2, we 279 used MSMD combined with a QM/MM scheme. The FEP was 280 calculated for the 2P-ERK2-ATP-substrate system described in 281 the ERK2 Starting Structures subsection and two additional 282 systems: 2P-ERK2 K52A-ATP-substrate and OP-ERK2-ATP283 (MSII)-substrate. The former was built by mutating the 284 conserved Lys52 to alanine in the 2P-ERK2-ATP-substrate 285 system and the latter by removing the Mg ion (MSI site) from 286 a OP-ERK2-ATP structure (derived from the MDs) and adding 287 the tripeptide substrate as previously described.

288 QM/MM Methods. The system was divided into a classical 289 part (MM) and a small quantum part (QM). The QM 290 subsystem consisted of the triphosphate part of ATP, the Mg 291 cation(s), the closest water molecules completing the 292 hydration shell of the ions, and the side chain of the catalytic 293 pocket residues comprised by Asp147, Asn152, Asp165 with 294 the standard protonation state. We also include the side chain 295 of the Thr of the target peptide reaching a total of 34 atoms for 296 the 2P-ERK2-ATP-substrate system (60 if all hydrogen atoms are included); the self-consistent charge density functional 297 tight binding (SCC-DFTB ${ }^{49}$ level of theory was used, offering 298 a balanced trade-off between accuracy and computational cost 299 (implemented in AMBER 16 package ${ }^{27}$ ). The rest of the ERK2 300 protein (classical subsystem), together with the surrounding 301 water molecules, were treated classically with the AMBER14SB 302 force field and the above-mentioned parameters for modified 303 amino acids. All simulations were performed with periodic 304 boundary conditions, a time step of $1 \mathrm{fs}$, an electrostatic cutoff 305 of $15 \AA$, and a total production time length of 100 ps. The 306 interface between the $\mathrm{QM}$ and $\mathrm{MM}$ portions was treated 307 accordingly with the link atom method as implemented in the 308 sander module. ${ }^{49}$

MSMD Strategy. The MSMD method ${ }^{50-53}$ was successfully 310 used previously by our group ${ }^{50-52,54}$ and comprises the use of 311 a set of nonequilibrium pulling trajectories to obtain the 312 associated free energy profile $(\Delta G)$. Briefly, in each trajectory, 313 the system is conducted through a reaction coordinate (RC) 314 by applying a time-dependent potential. $H(r, \lambda)$ is the 315 Hamiltonian of a system that is subject to an external time- 316 dependent potential $(\lambda=\lambda(t)) . \Delta G(\lambda)$ and $W(\lambda)$ are the 317 change in free energy and the external work performed on the 318 system as it evolves from $\lambda=\lambda_{0}$ to $\lambda_{t}$, respectively. The external 319 work is performed by the guiding or steering force. Here $r 320$ depicts a configuration of the whole system, while $\lambda$ is the 321 chosen reaction coordinate. Then, $\Delta G(\lambda)$ and $W(\lambda)$ are 322 related to each other by the following equation, known as 323 Jarzynski's relationship $^{55}$ (eq 2).

$$
\mathrm{e}^{-\beta \Delta G(\lambda)}=\left\langle\mathrm{e}^{-\beta W(\lambda)}\right\rangle
$$

The brackets in eq 2 represent an average taken over an 326 ensemble of molecular dynamics trajectories provided the 327 initial ensemble is equilibrated. Thus, in practice, in order to 328 obtain $\Delta G(\lambda)$, multiple trajectories are performed in which the 329 system is steered from reactants to products along $\lambda$, using an 330 external force (which usually takes a harmonic potential form) 331 and the work $(\lambda)$ performed is measured along the trajectory. 332 Once several trajectories and the corresponding work $(\lambda) 333$ profiles have been determined, the free energy profile $G(\lambda)$ is 334 obtained using eq 2 . To perform each trajectory, equilibrated 335 snapshots were randomly taken from classical Molecular 336 Dynamics simulations with a separation between frames of 337 no less than $5 \mathrm{~ns}$, beginning after the first $50 \mathrm{~ns}$ of the 338 corresponding classical MD simulations. We use a Born- 339 Oppenheimer molecular dynamics simulation to equilibrate 340 the system for 25 ps. Then, we selected a combination of four 341 distances as the reaction coordinate, as described in eq 3: 342

$$
\begin{aligned}
\lambda(r)= & d(\mathrm{O} 3 \beta-\mathrm{P} \gamma)-d(\mathrm{P} \gamma-\mathrm{O} \gamma)+d(\mathrm{O} \gamma-\mathrm{H} \gamma) \\
& -d(\mathrm{H} \gamma-\mathrm{O} 2 \delta)
\end{aligned}
$$

This reaction coordinate allows a joint description of the 344 phosphate transfer, characterized by the breaking of the $\mathrm{O} 3 \beta-345$ $\mathrm{P} \gamma$ bond in the ATP, and formation of the $\mathrm{P} \gamma-\mathrm{O} \gamma$ bond 346 between the gamma phosphate and the $\mathrm{OH}$ of the substrate. 347 As well as the proton transfer of the attacking nucleophile to 348 the Asp 147 acid acting as the acceptor base, and characterized 349 by the breaking of the $\mathrm{O} \gamma-\mathrm{H} \gamma$ bond, and formation of the 350 Asp 147:O $\delta-\mathrm{H} \gamma$ bond. In our particular system, the starting 351 points of the RC were $-4.0,-4.0$, and $-6.0 \AA$ for the $2 \mathrm{P}-352$ ERK2-ATP-substrate, 2P-ERK2 K52A-ATP-substrate, and 0P- 353 ERK2-ATP-substrate, respectively. 
A

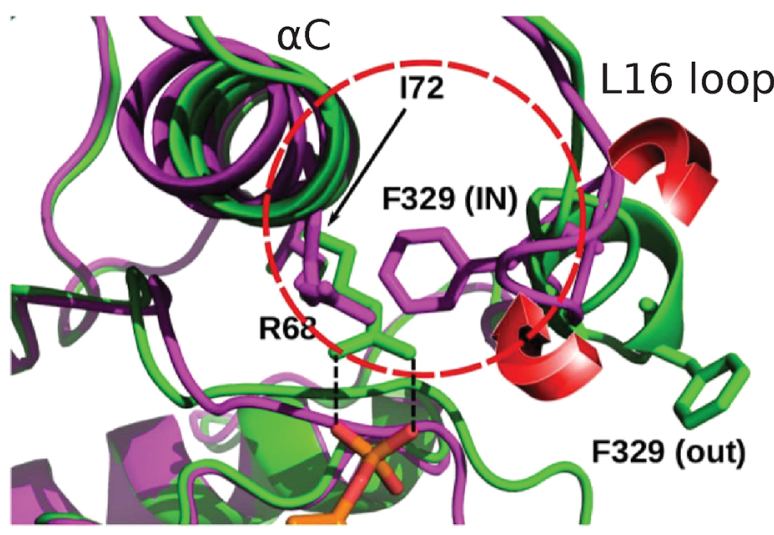

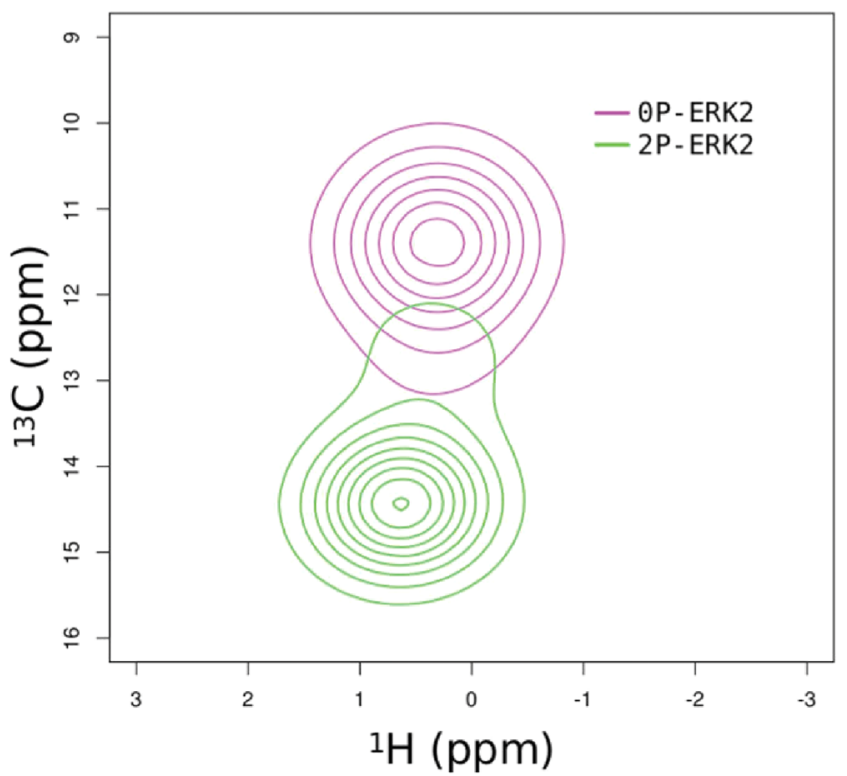

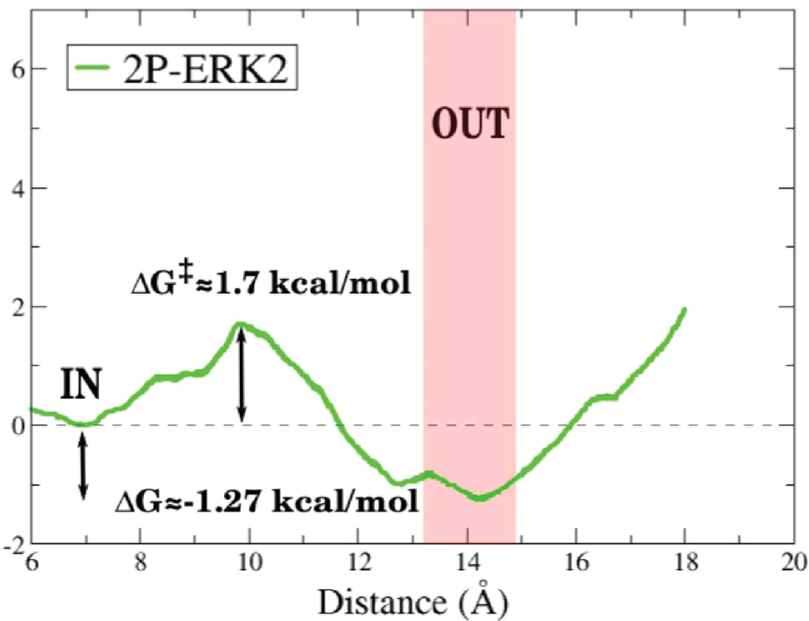

Figure 2. Phe329 as a reporter of the conformational change. (A) Shell of Ile72 (red circle), the principal conformational change involves Phe329 rotation. The red arrows shows the direction of the 3/10 helix that regulates the Phe-IN/Phe-OUT conformation. (B) Computed HMQC peaks of OP-ERK2 and 2P-ERK2 at $25{ }^{\circ} \mathrm{C}$. (C) Free energy profile for folding-unfolding of the L16 loop using the distance between Ile72 (C $\left.\alpha\right)$ and Phe329 (C $\alpha$ ). Unphosphorylated ERK2 (OP-ERK2) and dual-phosphorylated ERK2 (2P-ERK2) are colored magenta and green, respectively.

355 For all MSMD simulations, a pulling speed of $0.07 \AA /$ ps was 356 used. Faster speeds were tested but resulted in too much 357 variation in the $W(\lambda)$ profiles, thus leading to convergence 358 problems of the potential of mean force (PMF). The number 359 of MSMD runs used to determine each PMF varied in the 1036030 range as described in the results section, showing a good 361 convergence for a small number of curves (Figure S6). The 362 convergence of PMF and the standard deviation of each point 363 of the profile was determined with a leave-one-out cross364 validation methodology.

\section{$365 \square$ RESULTS}

366 ERK2 Undergoes a Small Conformational Rearrange367 ment upon Phosphorylation. We begin our analysis 368 looking in detail at ERK2 structural changes upon phosphor369 ylation (Figure1A) and its comparison with four well-studied 370 protein kinases (CDK2, PKA, EGFR, and SRC) (Figure1B,C). 371 Although in ERK2 the activation loop significantly rearranges when phosphorylated, there is not a significant reorientation of 372 the $\mathrm{N}$ and C-terminal lobes, in contrast to what is observed for 373 other PKs. Usually, in other kinases this change involves a 374 rotation of the $\alpha \mathrm{C}$-helix, with the concomitant movement of 375 the beta sheets and the glycine-rich loop of the N-terminal 376 lobe, a movement that can be characterized by the angle 377 between the $\alpha \mathrm{C}$-helix and $\alpha \mathrm{F}$-helix or the interlobe rotation 378 (see Methods) (Figure 1A,B and Figure S7). PKA has the 379 largest rotation angle, $18^{\circ}$, if the unphosphorylated enzyme 380 (PDB ID 4DFY) is compared with the active apoenzyme 381 (PDB ID 1J3H) or an even larger angle of $26^{\circ}$ when compared 382 with the active holoenzyme (PDB ID 1ATP). ${ }^{37}$ The rotation is 383 similar in EGFR upon activation; SRC has a similar angle, but 384 the rotation is in the opposite direction, while in CDK2 the 385 angle is around $10^{\circ}$. However, surprisingly ERK2 crystallo- 386 graphic structures show a rotation of only $5^{\circ}$ as described by 387 Barr $^{13}$ (Figure 1B,C). Consistently, the N-terminal lobe 388 RMSD between active and inactive structures determined 389 

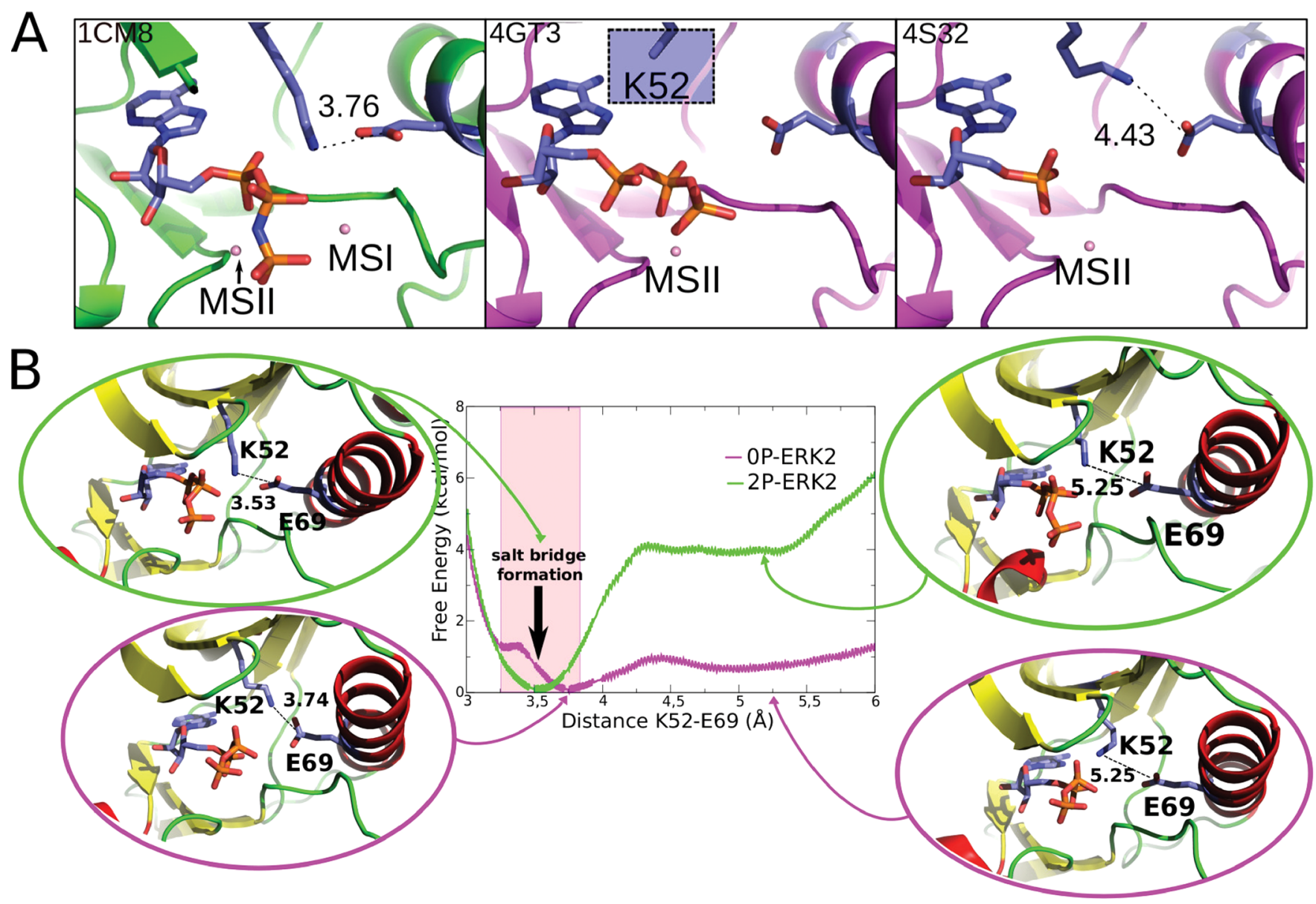

Figure 3. Salt bridge formation between Lys52 and Glu69. (A) Representative crystallographic structures of MAPKs. 2P-P38 $\gamma$ (PDB ID: 1CM8; left), 0P-ERK2 (PDB ID: 4GT3, 4S32; center and right). The ligands are respectively ANP, ATP, and ANP (P- $\beta$ and P- $\gamma$ coordinates were not determined). The blue square (middle panel) highlights that for the Lys52 residue the side-chain is not resolved in the X-ray structure, probably due to its flexibility. (B) Free energy profile for salt-bridge formation. 0P-ERK2-ATP and 2P-ERK2-ATP are colored magenta and green, respectively. The $\mathrm{Mg}$ ions are not shown.

390 after fitting the C-terminal lobe is $2 \AA$ in PKA but less than 0.7 $391 \AA$ in ERK2 (Figure 1C). Another characteristic that has been 392 used to describe kinase activation is the configuration of the so393 called R (regulatory) and C (catalytic) spine residue 394 interactions (Figure S8) ) $^{56-58}$ which are disrupted in the 395 inactive state and formed in the active state. However, in ERK2 396 we observed no significant differences in the configuration 397 between the inactive and phosphorylated protein for these 398 residues. Thus the spine is formed in both states. These results 399 are consistent with the fact that the structure-based 400 classification of PKs in the active or inactive states has failed 401 to correctly assign ERK2 structures $^{59}$ and suggests these 402 changes could be necessary but are not sufficient to control 403 enzymatic activity.

404 Previous studies ${ }^{14}$ also proposed that ERK2 may undergo a 405 conformational change in solution that is different from that 406 observed in the crystal structures. Therefore, and to analyze 407 the role of ERK2 conformational dynamics with the activation 408 process, we performed long $(1 \mu$ s per replica $)$ classical MD 409 simulations of both 0P-ERK2 and 2P-ERK2. Results show that 410 both states oscillate around the observed crystal (initial) 411 structure and no major changes are observed. Consistently, a 412 significant overlap of the rotational angle histogram was 413 observed between the active and inactive states either with or 414 without ATP bound (OP-ERK, 2P-ERK, OP-ERK-ATP, and
2P-ERK-ATP), indicating that the conformational change is 415 subtle and that both states are mutually visited (Figure 1B and 416 Figure S7). The small change observed for the conformational 417 angle, (of ca. 5 degrees between OP and 2P-ERK2 X-ray 418 structures) is also in sharp contrast to what is observed in other 419 PKs where the change is significantly larger ( $>10$ degrees). 420 Similar results are obtained when ERK2 dynamics are analyzed 421 using Essential Modes (Figure S9), since the projection of both 422 trajectories on the inactive-to-active transition mode (see 423 Methods), shows significant overlap, underscoring the fact that 424 both conformations of the kinase domain are mutually visited. 425 These results together with the similarity in the X-ray 426 structures, ${ }^{6,10}$ suggest that inactive and active ERK2 domain 427 conformations are not only similar but are mutually visited 428 during long protein dynamics in solution. Even though $1 \mu \mathrm{s} 429$ molecular dynamics are not able to sample larger configura- 430 tional changes that occur on the milliseconds to seconds scale, 431 we hypothesize that the small conformational change observed 432 in ERK2, compared to the other PKs, has a significant barrier 433 responsible for ERK2 phosphorylation-dependent activation. 434 This could explain that previous NMR studies showed two 435 conformations in solution. ${ }^{14}$

436

Conformational Change in the L16 Loop. A previous 437 study by Xiao and collaborators ${ }^{14}$ monitored aliphatic side 438 chains of Ile, Val, and Leu residues using NMR spectroscopy 439 


\section{Transition State}

A

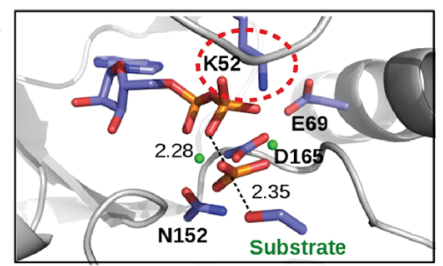

FreeEnergy profile

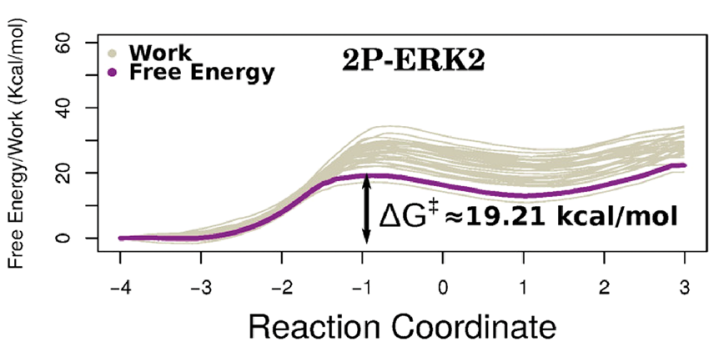

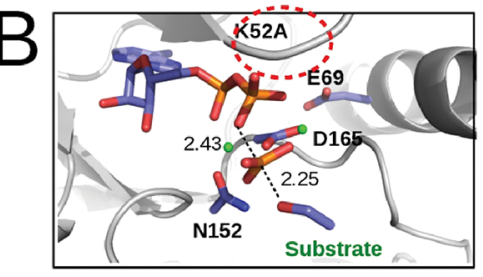

C

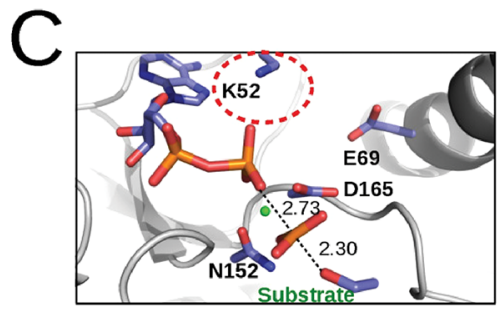

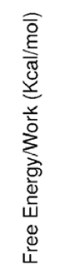
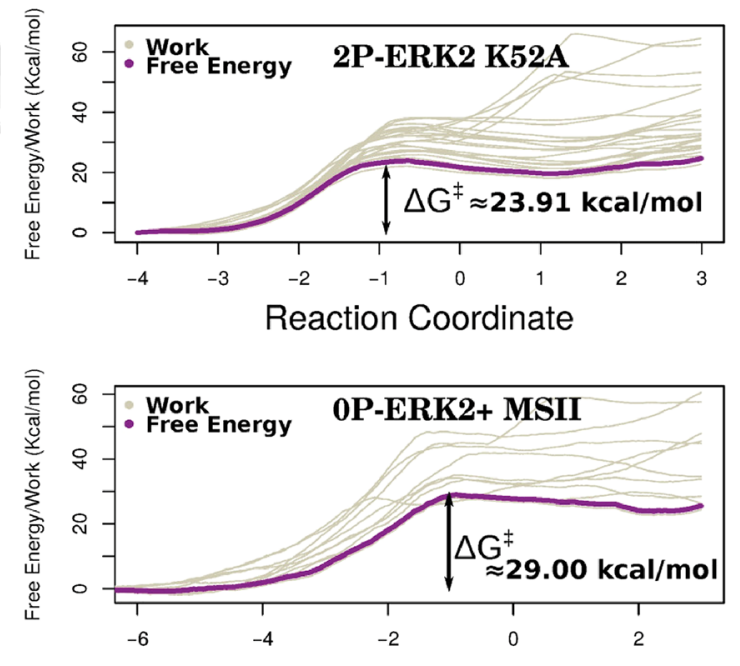

Reaction Coordinate
Associativity

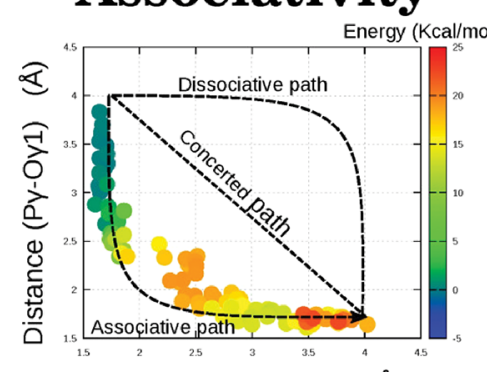

Distance (O3ß-Py) ( $(\AA)$

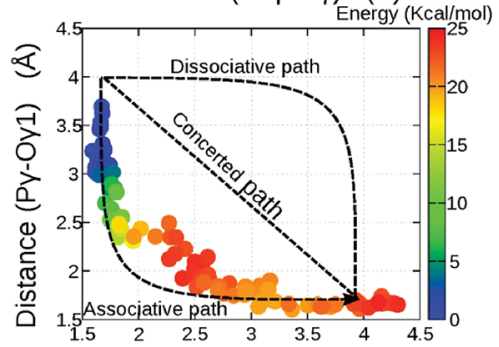

Distance (O3ß-PY) (A)

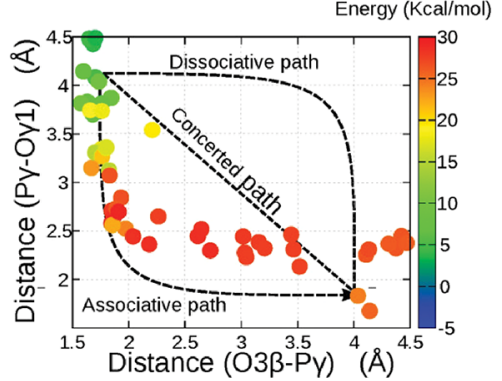

Figure 4. Free energy profile and structural parameters of the phosphotransfer reaction in ERK2. The mechanism of the phosphorylation process was evaluated and characterized in the 2P-ERK2-ATP (A), 2P-ERK2-ATP K52A mutant (B), and OP-ERK2-ATP (without the MgI ion) (C) states. For each setup we show the obtained transition state (left panel), the work for each run, and the global free energy (middle panel depicted in gray and purple, respectively). Finally, for each state an associativity plot, $\mathrm{P} \gamma / \mathrm{O} 3 \beta$ (substrate bond) vs $\mathrm{P} \gamma / \mathrm{O} \gamma 1$ (product bond) distances, was computed (right panel), showing the corresponding free energy values in a color scale. The $\mathrm{Mg}$ ions are shown as green dots.

440 and showed that while OP-ERK2 exhibits a unique con441 formation, 2P-ERK2 dynamics was fitted to a two-state model 442 with a $20 \%$ population of state- 1 - presumably inactive $0 \mathrm{P}$ 443 ERK2-like-and $80 \%$ of state-2 which would be the bio444 logically active state. ${ }^{14}$

445 Ile72, located in a central position of the $\alpha \mathrm{C}$ helix, showed a 446 significant change in chemical shift and also had a relationship 447 between delta-omega (CPMG chemical shift) and $k_{\text {ex }}$ that 448 reflected a slow exchange on the NMR chemical shift time 449 scale $\left(k_{\text {ex }} \ll\right.$ delta-omega $)$, which resulted in two distinct 450 peaks, corresponding to each population. In the inactive 451 structure, the Phe 329 (located in the L16 loop) interacts with 452 Ile $72 \delta$-carbon and will thus be referred to the Phe-IN state. 453 On the contrary, in the 2P-ERK2 X-ray structure, Ile72 side 454 chain is exposed to the solvent since the L16 loop Phe329 ring 455 is extended outward. We called that conformation the Phe456 OUT state (Figure 2A). We hypothesized that the observed 457 NMR change and the calculated populations could be due to 458 the small conformational change observed in the L16 loop, due 459 to the flipping of Phe329 from the Phe-IN to the Phe-OUT 460 state.

461 To support our hypothesis that this Phe-IN to Phe-OUT 462 change is partially responsible for the experimental observa463 tions, we first computed the Ile $72 \mathrm{H}-{ }^{13} \mathrm{C}$ NMR shifts in both 464 conformations using SHIFT2X. ${ }^{60}$ Our results, presented in
Figure $2 \mathrm{~B}$, are in qualitative agreement with the experimental 465 observations, ${ }^{14}$ showing the visible presence of two peaks. 466 Second, we computed the free energy profile for the Phe-IN to 467 Phe-OUT conformational transition using umbrella sampling 468 (US) with the distance between Ile72 and Phe329 as the 469 reaction coordinate, presented in Figure 2C. The resulting free 470 energy difference between both conformations is remarkably 471 consistent with the experimentally obtained populations: $\Delta G^{\circ} 472$ $=3.10 \mathrm{kcal} / \mathrm{mol}$ for OP-ERK2 and $\Delta G^{\circ}=-0.80 \mathrm{kcal} / \mathrm{mol}$ for 473 2P-ERK2. ${ }^{14}$ The profile also shows that in 2P-ERK2 there is a 474 relatively small barrier between both conformations $(\sim 1.7475$ $\mathrm{kcal} / \mathrm{mol}$ ), while the inactive ERK2 moving to the Phe-OUT 476 state has a barrier of $\sim 5.5 \mathrm{kcal} / \mathrm{mol}$. These results strongly 477 suggest that the NMR observed configurations and their 478 populations could be explained by the small rearrangement of 479 the L16 loop.

ERK2 Phosphorylation Stabilizes the Conserved Salt 481 Bridge between Lys52 and Glu69. To understand how 482 ERK2 phosphorylation promotes its activation, we measured 483 the free energy profile of a key salt bridge between Lys52 from 484 strand $\beta 3$ and Glu 69 from $\alpha \mathrm{C}$ helix in the holo (with ATP and 485 $2 \mathrm{Mg}$ ions) forms of the active and inactive states (OP-ERK2- 486 ATP and 2P-ERK-ATP) (Figure 3A). This salt bridge is highly $487 \mathrm{f3}$ conserved in the kinase family and is considered a hallmark of 488 
489 the activated state. ${ }^{56}$ In fact, it has been shown to be a great 490 classifier of kinase conformations. ${ }^{59}$

491 Figure 3B presents the classical free energy profile for 492 breaking the salt bridge in both the OP-ERK2-ATP and 2P493 ERK2-ATP states. The curves were obtained by performing US 494 simulations (see Methods) starting with the salt bridge formed 495 at a Lys52: $\epsilon-\mathrm{NH}_{3}^{+}-\mathrm{Glu} 69: \mathrm{C} \delta$ distance of $3.5 \AA$ and broken at $4965.3 \AA$. The results clearly show that for inactive ERK2 (0P497 ERK2-ATP) the salt bridge interaction free energy is minimal, 498 since breaking it requires $1 \mathrm{kcal} / \mathrm{mol}$, and there is a shallow 499 minimum at ca. 5.2 $\AA$. This result is indeed in agreement with 500 the conformation observed in the inactive complexes (PDB 501 IDs 4GT3 and 4S32). The first complex lacks the three distal 502 atoms on the side chain as expected if a residue has increased 503 flexibility, and in the second one the distance between Lys52 504 and Glu69 is $4.4 \AA$ (Figure 3A). On the contrary, breaking this 505 interaction in the $2 \mathrm{P}$-ERK2-ATP complex requires $4 \mathrm{kcal} / \mathrm{mol}$, 506 and there is no other clear minimum. In other words, in the 507 active state the salt bridge is established, while it is labile in the 508 inactive state.

\section{Impact of Lys52-Glu69 Salt Bridge Formation on the} 510 Phosphate Transfer Mechanism. To evaluate the impact of 511 the Lys52-Glu69 salt bridge on the phosphate transfer 512 mechanism we first analyzed how it affects the $\mathrm{Mg}$ affinity. 513 The effects of $\mathrm{Mg}$ binding (MSI) for all protein states were 514 calculated according to the thermodynamic cycles shown in 515 Figure S5. Interestingly, the presence of the salt bridge 516 increases the $\mathrm{MgI}$ affinity in $8.6 \mathrm{kcal} / \mathrm{mol}$, and most 517 importantly, it retains the ATP-P $\gamma$ and the $\mathrm{MgI}$ in a proper 518 "reactive" configuration. It is important to remark that this 519 result qualitatively highlights that there is a correlation 520 between the salt bridge formation and the affinity for the $521 \mathrm{MSI}$ Mg cation. This is in agreement with the experimental 522 results. In the $0 \mathrm{P}-\mathrm{ERK} 2$ state for the available X-ray structures 523 (4GT3 and 4S32), the MgI is absent and the atoms involved in 524 the interaction either are missing or too far. Supporting this 525 hypothesis, the molecular dynamics of OP-ERK2-ATP exhibit 526 an incipient break of the coordination sphere of the MSI site, 527 mainly due to the proximity of Glu69 to the $\mathrm{Mg}$ ion (Figure 528 S10).

529 Second, and most important, we analyzed its effect on the 530 energetics and mechanism of the phosphate transfer step, by 531 computing with multiscale QM/MM MD simulations the 532 corresponding free energy profiles using a tripeptide with a Thr 533 phosphoacceptor as substrate. Figure 4 presents the corre534 sponding results for the reactive conformation (2P-ERK2-ATP 535 with the Lys52-Glu69 salt bridge properly established, Figure $5364 \mathrm{~A}$ ), the same structure but mutating Lys52 for Alanine, thus 537 removing the salt bridge (Figure 4B), and the conformation 538 reached after losing the $\mathrm{Mg}$ ion in the MSI site (Figure 4C) of 539 the OP-ERK2-ATP state. Consistent with our hypothesis, 540 breaking the Lys52-Glu69 interaction results in a small but 541 significant increase in the reaction barrier (from 19.2 to 23.9 $542 \mathrm{kcal} / \mathrm{mol}$ ), and loosing the $\mathrm{MgI}$ in the unphosphorylated form 543 raises it even more, to $29.0 \mathrm{kcal} / \mathrm{mol}$.

544 Concerning the reaction mechanism, as evidenced in the 545 projection of the reaction coordinate onto the $2 \mathrm{D} \mathrm{RC}$ plots 546 (Figure 4, right panels), in all cases the reaction displays mostly 547 an associative character. The phosphoacceptor must first come 548 from its equilibrium distance to about $2.5 \AA$ before the $\mathrm{O} 3 \beta-\mathrm{P} \gamma$ 549 bond of the ATP starts to break. Interestingly, in the transition 550 state (TS) region the reaction becomes dissociative-like, with 551 the $\mathrm{O} 3 \beta$ - $\mathrm{P} \gamma$ distance increasing from ca. 1.8 to $2.5 \AA$ reaching the TS structure with a trigonal planar transferring phosphate, 552 a geometry that has also been observed in crystal structures of 553 other S/T kinases with transition state analogues. 40,61 $^{2}$

Comparatively, as the MSI site becomes disorganized when 555 the salt bridge is broken, and finally lost, the active site 556 becomes looser, a fact that is evidenced in the increasing 557 dispersion of the individual work profiles, which contribute to 558 an increase in the free energy barrier. Also interestingly, in the 559 TS region, the reaction becomes more dissociative-like. In fact, 560 in the absence of $\mathrm{Mg}^{2+}$ the substrate- $\mathrm{P} \gamma$ bond is more often 561 than not correctly established.

We propose that the $\mathrm{Mg}$ ions have a preponderant role in 563 the reaction catalyzed by ERK2, stabilizing the transition state. 564 Also, we have previously shown that a key step in the reaction 565 is the proton transfer from the substrate Thr to the side chain 566 of Asp147, which belongs to a highly conserved motif in the S/ 567 $\mathrm{T}$ kinases (HRD motif). ${ }^{17}$ In agreement, a similar behavior for 568 the 2P-ERK2-ATP state is observed (Figure S11, panel A). 569 While the active state presents a concurrent proton transfer 570 step, in the inactive state, the transfer occurs later (Figure S11, 571 panel C). We can also point out that in the inactive ERK2, the 572 coordination sphere of the MSII site changes while the 573 phosphotransfer reaction occurs (Figure S11 E,F). 574

\section{DISCUSSION}

575

Because of the enormous relevance of PKs, the underlying 576 structural reasons that characterize their phosphorylation- 577 dependent activation has been a significant area of research 578 during the last decades. Analysis of hundreds of different 579 active-inactive pairs of PKs structures yields several common 580 features that presently describe, and define, how a PK is 581 structurally activated. Phosphorylation in the activation 582 segment loops usually triggers a reorganization of the more 583 flexible C-terminal lobe, which rotates and closes with respect 584 to the N-terminal lobe, thereby correctly "shaping" the ATP 585 binding active site for catalysis. This closure is accompanied by 586 the formation of both hydrophobic spines, the so-called 587 Regulatory (R) and Catalytic (C) spines, which respectively 588 define the active state and catalytic competent active site. $\quad 589$

This conformational change is observed in several PKs, such 590 as PKA, CDK2, or Src. Indeed recent works in JNK3 and p38 591 (both representatives members of the MAPKs superfamily) 592 show the importance of the elucidation of the activation 593 mechanism to drug design. Although the works describe and 594 even identify possible intermediaries of the activation process, 595 they do not show a direct relationship between the 596 conformational change and the phosphorylation process. ${ }^{62,63} 597$ Also, we must consider that the hydrophobic spines in ERK2 598 are present in the inactive state, and thus other explanations 599 are needed, highlighting the plasticity of the kinase domain. $\quad 600$

In this work, we used multiscale free energy based molecular 601 simulations of ERK2 to determine the key structural and 602 dynamical features that explain how ERK2 phosphorylation is 603 coupled to an over 50000 times increase in its catalytic power. 604 Consistent with the structural data, our results show both that 605 inactive ERK2 and phosphorylated ERK2 display similar 606 conformations, except for that of the L16 loop, and both 607 states are mutually visited during equilibrium state molecular 608 dynamics. Moreover, no significant rotation of helix-C or 609 change in the size of the ATP binding pocket is observed. The 610 $\mathrm{R}$ and $\mathrm{C}$ spines remain in a similar state and most residues, 611 including those involved in catalysis, seem to be in a similar 612 configuration. Taking all this into account we conclude that 613 


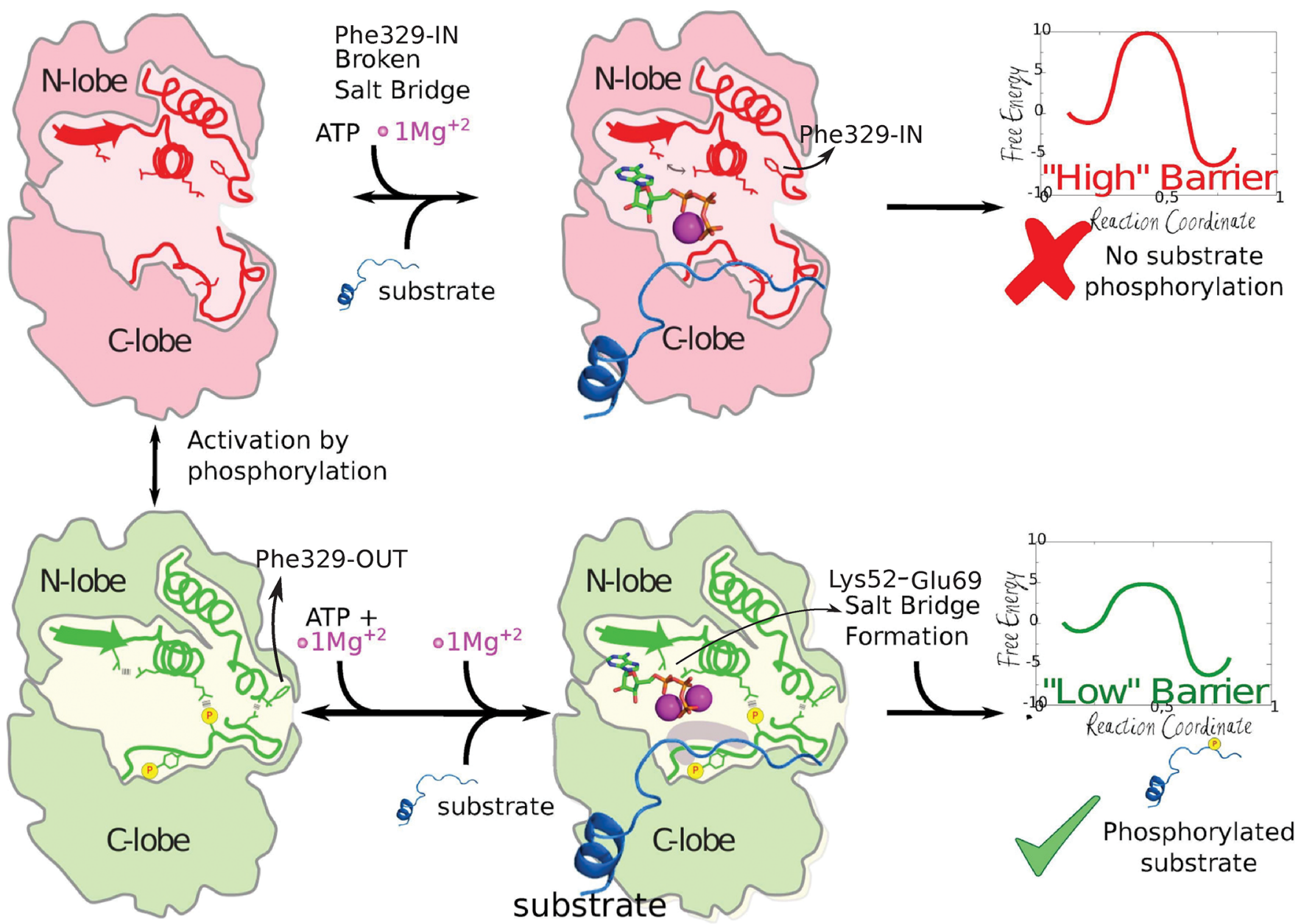

Figure 5. The activation of ERK2 kinase catalytic activity. Phosphorylation and reorganization of the activation loop promote kinase activation.

614 other, possibly smaller, but significant changes must regulate 615 ERK2 kinase activation.

616 The best possible candidate can relate to previous NMR 617 studies which showed the presence of two conformations (here 618 referred to as Phe-IN and Phe-OUT), that shift their 619 population upon phosphorylation. We were able to character620 ize this change at the atomic level, described mainly by the 621 rotation of the L16 loop with the concomitant solvent 622 exposure of Phe329 in the Phe-OUT state, which shifts the ${ }_{623}$ NMR signal of Ile72, as shown by Xiao et. al, ${ }^{14}$ and is the 624 preferred state in phosphorylated ERK2. Remarkably, even 625 though both the unphosphorylated and phosphorylated 626 configurations are similar, the latest state significantly enhances 627 the strength of the key Lys52-Glu69 (ERK2 numbering) salt 628 bridge. This interaction correlates with a stabilization of the $629 \mathrm{Mg}^{2+}$ binding site $\mathrm{I}^{15}$ and retains ATP in a reactive 630 conformation, therefore controlling the kinetic barrier of the 631 phosphate transfer step (as summarized in Figure 5). In 632 agreement with our results, recent work in PKA shows a 633 change in the dihedral angle population between both states, 634 for the same salt bridge. ${ }^{64}$ The fact that the nonphosphorylated 635 ERK2 structure (PDB ID 4GT3) lacks the cation in the MSI 636 site also supports our observation. Also interesting, the 637 structure of CDK2 transition-state-like complex (PDB ID 638 3QHR), suggest that phosphate transfer occurs through an 639 associative mechanism, as also displayed by our results for the
Phe-OUT state (2P-ERK2), a characteristic that is partially lost 640 in the Phe-IN state (OP-ERK).

A relevant role for the interaction between the conserved 642 Lys52 and Glu69 residues has been previously highlighted for 643 other kinases. However, it has been mostly seen in the context 644 of the above-mentioned major configurational rearrangement 645 and not as the key factor governing activation. ${ }^{64-66} 646$ Phosphorylation of the activation loop stabilizes the Phe- 647 OUT conformation and stabilizes the salt bridge necessary for 648 proper catalysis. In agreement with our results, previous studies 649 in Hog1 and p38 $\alpha^{67-69}$ have shown that mutation of the 650 conserved Phe residue to Leu in the L16 loop, which promotes 651 stabilization of the Phe-OUT state, produces intrinsically active 652 mutants and promotes dimerization. It has also been proposed 653 that dimerization of ERK2, which has been linked to the Phe- 654 OUT state, enhances catalytic activity and is essential for 655 nuclear translocation. ${ }^{6}$

656

Taking our results in the context of previous works 657 concerning the relationship between phosphorylation-depend- 658 ent conformational changes and catalytic activity in PKs, we 659 can argue that they employ different inactivation mechanisms, 660 as already indicated by other authors. ${ }^{57,70}$ The assembly of the 661 hydrophobic $\mathrm{R}$ and $\mathrm{C}$ spines with domain rotation and closure 662 are important in several cases, but it must be considered that to 663 increase the catalytic power, and thus kinase activity, these 664 changes must result in either higher affinity for the substrates 665 (ATP-phosphoacceptor) and/or lower barrier for the chemical 666 
667 step. Our results show that despite being small, the 668 conformational change in ERK2 triggered by dual-phosphor669 ylation results in the stabilization of the "biologically active" 670 Phe-OUT state, increased binding of the $\mathrm{MgI}$ ion, and the 671 formation of a key salt bridge.

\section{$672 \square$ ASSOCIATED CONTENT}

673 S Supporting Information

674 The Supporting Information is available free of charge on the 675 ACS Publications website at DOI: 10.1021/acs.jcim.9b00782.

676 OP-ERK2 and 2P-ERK2MD metrics and essential modes 677 projection, $\mathrm{Mg}$ ion coordination information, $\mathrm{C}$ and $\mathrm{R}$ 678 spine evaluation, US and MSMD validation (PDF)

679 Coordinates of the four ERK2 initial models (ZIP)

\section{AUTHOR INFORMATION}

\section{Corresponding Author}

682 E-mail: adrian@qb.fcen.uba.ar.

683 ORCID

684 Elias D. López: 0000-0002-9956-0010

685 Osvaldo Burastero: 0000-0003-4089-0434

686 Juan Pablo Arcon: 0000-0003-3350-1576

687 Lucas A. Defelipe: 0000-0001-7859-7300

688 Marcelo A. Marti: 0000-0002-7911-9340

689 Adrian G. Turjanski: 0000-0003-2190-137X

690 Notes

691 The authors declare no competing financial interest.

\section{ACKNOWLEDGMENTS}

693 The authors thank Mehrnoosh Arrar for useful discussions. 694 Computer time was provided by Centro de Computos de Alto 695 Rendimiento (CeCAR), FCEN-UBA and TUPAC-CSC696 CONICET. E.D.L., J.P.A., and L.A.D. acknowledge ANPCyT 697 and CONICET, respectively, for postdoctoral fellowship. O.B. 698 acknowledges ANPCyT and CONICET for doctoral fellow699 ship. L.A.D., M.A.M., and A.G.T. are members of the 700 CONICET. This work was supported by grants of ANPCyT 701 (PICT 2015-2276) awarded to A.G.T.

\section{$702 \square$ REFERENCES}

703 (1) Huse, M.; Kuriyan, J. The conformational plasticity of protein 704 kinases. Cell 2002, 109, 275-282.

705 (2) Hoshino, R.; Chatani, Y.; Yamori, T.; Tsuruo, T.; Oka, H.; 706 Yoshida, O.; Shimada, Y.; Ari-i, S.; Wada, H.; Fujimoto, J.; Kohno, M. 707 Constitutive activation of the $41-/ 43-\mathrm{kDa}$ mitogen-activated protein 708 kinase signaling pathway in human tumors. Oncogene 1999, 18, 813. 709 (3) Gioeli, D.; Mandell, J. W.; Petroni, G. R.; Frierson, H. F.; Weber, 710 M. J. Activation of mitogen-activated protein kinase associated with 711 prostate cancer progression. Cancer Res. 1999, 59, 279-284.

712 (4) Lawrence, M. C.; Jivan, A.; Shao, C.; Duan, L.; Goad, D.; 713 Zaganjor, E.; Osborne, J.; McGlynn, K.; Stippec, S.; Earnest, S.; Chen, 714 W.; Cobb, M. H. The roles of MAPKs in disease. Cell Res. 2008, 18, 715436.

716 (5) Uehling, D. E.; Harris, P. A. Recent progress on MAP kinase 717 pathway inhibitors. Bioorg. Med. Chem. Lett. 2015, 25, 4047-4056.

718 (6) Canagarajah, B. J.; Khokhlatchev, A.; Cobb, M. H.; Goldsmith, 719 E. J. Activation mechanism of the MAP kinase ERK2 by dual 720 phosphorylation. Cell 1997, 90, 859-869.

721 (7) Robinson, M. J.; Harkins, P. C.; Zhang, J.; Baer, R.; Haycock, J. 722 W.; Cobb, M. H.; Goldsmith, E. J. Mutation of position 52 in ERK2 723 creates a nonproductive binding mode for adenosine 5 -triphosphate. 724 Biochemistry 1996, 35, 5641-5646.
(8) Gonzalez, F. A.; Raden, D. L.; Davis, R. J. Identification of 725 substrate recognition determinants for human ERK1 and ERK2 726 protein kinases. J. Biol. Chem. 1991, 266, 22159-22163. 727

(9) Remenyi, A.; Good, M. C.; Lim, W. A. Docking interactions in 728 protein kinase and phosphatase networks. Curr. Opin. Struct. Biol. 729 2006, 16, 676-685.

730

(10) Zhang, F.; Strand, A.; Robbins, D.; Cobb, M. H.; Goldsmith, E. 731 J. Atomic structure of the MAP kinase ERK2 at $2.3 \AA$ resolution. 732 Nature 1994, 367, 704.

(11) Cobb, M. H.; Goldsmith, E. J. Dimerization in MAP-kinase 734 signaling. Trends Biochem. Sci. 2000, 25, 7-9. 735

(12) Nguyen, T.; Ruan, Z.; Oruganty, K.; Kannan, N. Co-conserved 736 MAPK features couple D-domain docking groove to distal allosteric 737 sites via the C-terminal flanking tail. PLoS One 2015, 10, 738 No. e0119636.

739

(13) Barr, D.; Oashi, T.; Burkhard, K.; Lucius, S.; Samadani, R.; 740 Zhang, J.; Shapiro, P.; MacKerell, A. D., Jr; van der Vaart, A. 741 Importance of domain closure for the autoactivation of ERK2. 742 Biochemistry 2011, 50, 8038-8048.

(14) Xiao, Y.; Lee, T.; Latham, M. P.; Warner, L. R.; Tanimoto, A.; 744 Pardi, A.; Ahn, N. G. Phosphorylation releases constraints to domain 745 motion in ERK2. Proc. Natl. Acad. Sci. U. S. A. 2014, 111, 2506-2511. 746

(15) Bao, Z. Q.; Jacobsen, D. M.; Young, M. A. Briefly bound to 747 activate: transient binding of a second catalytic magnesium activates 748 the structure and dynamics of CDK2 kinase for catalysis. Structure 749 2011, 19, 675-690.

(16) Madhusudan; Akamine, P.; Xuong, N.-H.; Taylor, S. S. Crystal 751 structure of a transition state mimic of the catalytic subunit of cAMP- 752 dependent protein kinase. Nat. Struct. Mol. Biol. 2002, 9, 273.

(17) Turjanski, A. G.; Hummer, G.; Gutkind, J. S. How mitogen- 754 activated protein kinases recognize and phosphorylate their targets: A 755 QM/MM study. J. Am. Chem. Soc. 2009, 131, 6141-6148. 756

(18) Sours, K. M.; Xiao, Y.; Ahn, N. G. Extracellular-regulated kinase 757 2 is activated by the enhancement of hinge flexibility. J. Mol. Biol. 758 2014, 426, 1925-1935.

(19) Prowse, C. N.; Lew, J. Mechanism of activation of ERK2 by 760 dual phosphorylation. J. Biol. Chem. 2001, 276, 99-103. 761

(20) Hari, S. B.; Merritt, E. A.; Maly, D. J. Conformation-selective 762 ATP-competitive inhibitors control regulatory interactions and 763 noncatalytic functions of mitogen-activated protein kinases. Chem. 764 Biol. 2014, 21, 628-635.

(21) Smith, G. K.; Ke, Z.; Guo, H.; Hengge, A. C. Insights into the 766 phosphoryl transfer mechanism of cyclin-dependent protein kinases 767 from ab initio QM/MM free-energy studies. J. Phys. Chem. B 2011, 768 $115,13713-13722$.

(22) De Vivo, M.; Cavalli, A.; Carloni, P.; Recanatini, M. 770 Computational study of the phosphoryl transfer catalyzed by a 771 cyclin-dependent kinase. Chem. - Eur. J. 2007, 13, 8437-8444. 772 (23) Cheng, Y.; Zhang, Y.; McCammon, J. A. How does the cAMP- 773 dependent protein kinase catalyze the phosphorylation reaction: an ab 774 initio QM/MM study. J. Am. Chem. Soc. 2005, 127, 1553-1562. 775

(24) Montenegro, M.; Garcia-Viloca, M.; Lluch, J. M.; González- 776 Lafont, À. A QM/MM study of the phosphoryl transfer to the 777 Kemptide substrate catalyzed by protein kinase A. The effect of the 778 phosphorylation state of the protein on the mechanism. Phys. Chem. 779 Chem. Phys. 2011, 13, 530-539.

780

(25) Zhang, F.; Strand, A.; Robbins, D.; Cobb, M. H.; Goldsmith, E. 781 J. Atomic structure of the MAP kinase ERK2 at $2.3 \AA$ resolution. 782 Nature 1994, 367, 704.

(26) Bellon, S.; Fitzgibbon, M. J.; Fox, T.; Hsiao, H.-M.; Wilson, K. 784 $\mathrm{P}$. The structure of phosphorylated $\mathrm{p} 38 \gamma$ is monomeric and reveals a 785 conserved activation-loop conformation. Structure 1999, 7, 1057- 786 1065.

(27) Case, D. A.; Betz, R. M.; Cerutti, D. S.; Cheatham, T. E.; 788 Darden, T. A.; Duke, R. E.; Giese, T. J.; Gohlke, H.; Goetz, A. W.; 789 Homeyer, N.; Izadi, S.; Janowski, P.; Kaus, J.; Kovalenko, A.; Lee, T. 790 S.; LeGrand, S.; Li, P.; Lin, C.; Luchko, T.; Luo, R.; Madej, B.; 791 Mermelstein, D.; Merz, K. M.; Monard, G.; Nguyen, H.; Nguyen, H. 792 T.; Omelyan, I.; Onufriev, A.; Roe, D. R.; Roitberg, A.; Sagui, C.; 793 
794 Simmerling, C. L.; Botello-Smith, W. M.; Swails, J.; Walker, R. C.; 795 Wang, J.; Wolf, R. M.; Wu, X.; Xiao, L.; Kollman, P. A. AMBER 2016; 796 University of California, 2016.

797 (28) Jorgensen, W. L.; Chandrasekhar, J.; Madura, J. D.; Impey, R. 798 W.; Klein, M. L. Comparison of simple potential functions for 799 simulating liquid water. J. Chem. Phys. 1983, 79, 926-935.

800 (29) Maier, J. A.; Martinez, C.; Kasavajhala, K.; Wickstrom, L.; 801 Hauser, K. E.; Simmerling, C. ff14SB: improving the accuracy of 802 protein side chain and backbone parameters from ff99SB. J. Chem. 803 Theory Comput. 2015, 11, 3696-3713.

804 (30) Craft, J. W.; Legge, G. B. An AMBER/DYANA/MOLMOL 805 phosphorylated amino acid library set and incorporation into NMR 806 structure calculations. J. Biomol. NMR 2005, 33, 15-24.

807 (31) Meagher, K. L.; Redman, L. T.; Carlson, H. A. Development of 808 polyphosphate parameters for use with the AMBER force field. J. 809 Comput. Chem. 2003, 24, 1016-1025.

810 (32) Essmann, U.; Perera, L.; Berkowitz, M. L.; Darden, T.; Lee, H.; 811 Pedersen, L. G. A smooth particle mesh Ewald method. J. Chem. Phys. 812 1995, 103, 8577-8593.

813 (33) Berendsen, H. J.; Postma, J. v.; van Gunsteren, W. F.; DiNola, 814 A.; Haak, J. Molecular dynamics with coupling to an external bath. J. 815 Chem. Phys. 1984, 81, 3684-3690.

816 (34) Martyna, G. J.; Klein, M. L.; Tuckerman, M. Nosé-Hoover 817 chains: The canonical ensemble via continuous dynamics. J. Chem. 818 Phys. 1992, 97, 2635-2643.

819 (35) Hopkins, C. W.; Le Grand, S.; Walker, R. C.; Roitberg, A. E. 820 Long-time-step molecular dynamics through hydrogen mass reparti821 tioning. J. Chem. Theory Comput. 2015, 11, 1864-1874.

822 (36) Ryckaert, J.-P.; Ciccotti, G.; Berendsen, H. J. Numerical 823 integration of the cartesian equations of motion of a system with 824 constraints: molecular dynamics of n-alkanes. J. Comput. Phys. 1977, 825 23, 327-341.

826 (37) Steichen, J. M.; Kuchinskas, M.; Keshwani, M. M.; Yang, J.; 827 Adams, J. A.; Taylor, S. S. Structural basis for the regulation of protein 828 kinase A by activation loop phosphorylation. J. Biol. Chem. 2012, 287, $82914672-14680$.

830 (38) Akamine, P.; Madhusudan; Wu, J.; Xuong, N.-H.; Eyck, L. F.T.; 831 Taylor, S. S Dynamic features of cAMP-dependent protein kinase 832 revealed by apoenzyme crystal structure. J. Mol. Biol. 2003, 327, 159833171.

834 (39) Martin, M. P.; Olesen, S. H.; Georg, G. I.; Schonbrunn, E. 835 Cyclin-dependent kinase inhibitor dinaciclib interacts with the acetyl836 lysine recognition site of bromodomains. ACS Chem. Biol. 2013, 8, $8372360-2365$.

838 (40) Bao, Z. Q.; Jacobsen, D. M.; Young, M. A. Briefly bound to 839 activate: transient binding of a second catalytic magnesium activates 840 the structure and dynamics of CDK2 kinase for catalysis. Structure 841 2011, 19, 675-690.

842 (41) Zhang, X.; Gureasko, J.; Shen, K.; Cole, P. A.; Kuriyan, J. An 843 allosteric mechanism for activation of the kinase domain of epidermal 844 growth factor receptor. Cell 2006, 125, 1137-1149.

845 (42) Xu, W.; Doshi, A.; Lei, M.; Eck, M. J.; Harrison, S. C. Crystal 846 structures of c-Src reveal features of its autoinhibitory mechanism. 847 Mol. Cell 1999, 3, 629-638.

848 (43) Azam, M.; Seeliger, M. A.; Gray, N. S.; Kuriyan, J.; Daley, G. Q. 849 Activation of tyrosine kinases by mutation of the gatekeeper 850 threonine. Nat. Struct. Mol. Biol. 2008, 15, 1109.

851 (44) Rodriguez Limardo, R. G.; Ferreiro, D. N.; Roitberg, A. E.; 852 Marti, M. A.; Turjanski, A. G. P38 $\gamma$ activation triggers dynamical 853 changes in allosteric docking sites. Biochemistry 2011, 50, 1384-1395. 854 (45) Grossfield, A. WHAM: the weighted histogram analysis method, 855 version 2.0.9; University of Rochester Medical Centerhttp:// 856 membrane.urmc.rochester.edu/wordpress/?page $\backslash$ id $=126$.

857 (46) Kaus, J. W.; Pierce, L. T.; Walker, R. C.; McCammon, J. A. 858 Improving the efficiency of free energy calculations in the amber 859 molecular dynamics package. J. Chem. Theory Comput. 2013, 9, 41318604139.
(47) Steinbrecher, T.; Joung, I.; Case, D. A. Soft-core potentials in 861 thermodynamic integration: Comparing one-and two-step trans- 862 formations. J. Comput. Chem. 2011, 32, 3253-3263.

863

(48) Shirts, M. R.; Chodera, J. D. Statistically optimal analysis of 864 samples from multiple equilibrium states. J. Chem. Phys. 2008, 129, 865 124105.

866

(49) Cui, Q.; Elstner, M.; Kaxiras, E.; Frauenheim, T.; Karplus, M. A 867 $\mathrm{QM} / \mathrm{MM}$ implementation of the self-consistent charge density 868 functional tight binding (SCC-DFTB) method. J. Phys. Chem. B 869 2001, 105, 569-585.

870

(50) Crespo, A.; Martí, M. A.; Estrin, D. A.; Roitberg, A. E. Multiple- 871 steering QM- MM calculation of the free energy profile in chorismate 872 mutase. J. Am. Chem. Soc. 2005, 127, 6940-6941.

873

(51) Defelipe, L. A.; Lanzarotti, E.; Gauto, D.; Marti, M. A.; 874 Turjanski, A. G. Protein topology determines cysteine oxidation fate: 875 the case of sulfenyl amide formation among protein families. PLoS 876 Comput. Biol. 2015, 11, No. e1004051.

(52) Forti, F.; Boechi, L.; Estrin, D. A.; Marti, M. A. Comparing and 878 combining implicit ligand sampling with multiple steered molecular 879 dynamics to study ligand migration processes in heme proteins. J. 880 Comput. Chem. 2011, 32, 2219-2231.

881

(53) Xiong, H.; Crespo, A.; Marti, M.; Estrin, D.; Roitberg, A. E. 882 Free energy calculations with non-equilibrium methods: applications 883 of the Jarzynski relationship. Theor. Chem. Acc. 2006, 116, 338-346. 884

(54) Issoglio, F. M.; Campolo, N.; Zeida, A.; Grune, T.; Radi, R.; 885 Estrin, D. A.; Bartesaghi, S. Exploring the catalytic mechanism of 886 human glutamine synthetase by computer simulations. Biochemistry 887 2016, 55, 5907-5916.

(55) Jarzynski, C. Nonequilibrium equality for free energy 889 differences. Phys. Rev. Lett. 1997, 78, 2690.

(56) Taylor, S. S.; Kornev, A. P. Protein kinases: evolution of 891 dynamic regulatory proteins. Trends Biochem. Sci. 2011, 36, 65-77. 892 (57) Meharena, H. S.; Chang, P.; Keshwani, M. M.; Oruganty, K.; 893 Nene, A. K.; Kannan, N.; Taylor, S. S.; Kornev, A. P. Deciphering the 894 structural basis of eukaryotic protein kinase regulation. PLoS Biol. 895 2013, 11, No. e1001680.

(58) Kornev, A. P.; Taylor, S. S. Defining the conserved internal 897 architecture of a protein kinase. Biochim. Biophys. Acta, Proteins 898 Proteomics 2010, 1804, 440-444.

(59) McSkimming, D. I.; Rasheed, K.; Kannan, N. Classifying kinase 900 conformations using a machine learning approach. BMC Bioinf. 2017, 901 $18,86$.

(60) Han, B.; Liu, Y.; Ginzinger, S. W.; Wishart, D. S. SHIFTX2: 903 significantly improved protein chemical shift prediction. J. Biomol. 904 NMR 2011, 50, 43.

905

(61) Madhusudan; Akamine, P.; Xuong, N.-H.; Taylor, S. S. Crystal 906 structure of a transition state mimic of the catalytic subunit of cAMP- 907 dependent protein kinase. Nat. Struct. Mol. Biol. 2002, 9, 273.

(62) Mishra, P.; Günther, S. New insights into the structural 909 dynamics of the kinase JNK3. Sci. Rep. 2018, 8, 9435.

(63) Kuzmanic, A.; Sutto, L.; Saladino, G.; Nebreda, A. R.; Gervasio, 911 F. L.; Orozco, M. Changes in the free-energy landscape of p38 $\alpha$ MAP 912 kinase through its canonical activation and binding events as studied 913 by enhanced molecular dynamics simulations. Elife 2017, 6, 914 No. e22175.

915

(64) Ahuja, L. G.; Kornev, A. P.; McClendon, C. L.; Veglia, G.; 916 Taylor, S. S. Mutation of a kinase allosteric node uncouples dynamics 917 linked to phosphotransfer. Proc. Natl. Acad. Sci. U. S. A. 2017, 114, 918 E931-E940.

(65) Bastidas, A. C.; Deal, M. S.; Steichen, J. M.; Guo, Y.; Wu, J.; 920 Taylor, S. S. Phosphoryl transfer by protein kinase A is captured in a 921 crystal lattice. J. Am. Chem. Soc. 2013, 135, 4788-4798. 922

(66) Meharena, H. S.; Fan, X.; Ahuja, L. G.; Keshwani, M. M.; 923 McClendon, C. L.; Chen, A. M.; Adams, J. A.; Taylor, S. S. Decoding 924 the interactions regulating the active state mechanics of eukaryotic 925 protein kinases. PLoS Biol. 2016, 14, No. e2000127.

926

(67) Bell, M.; Capone, R.; Pashtan, I.; Levitzki, A.; Engelberg, D. 927 Isolation of hyperactive mutants of the MAPK p38/Hog1 that are 928 
929 independent of MAPK kinase activation. J. Biol. Chem. 2001, 276, $93025351-25358$.

931 (68) Diskin, R.; Askari, N.; Capone, R.; Engelberg, D.; Livnah, O. 932 Active mutants of the human $\mathrm{p} 38 \alpha$ mitogen-activated protein kinase. 933 J. Biol. Chem. 2004, 279, 47040-47049.

934 (69) Diskin, R.; Lebendiker, M.; Engelberg, D.; Livnah, O. 935 Structures of p38 $\alpha$ active mutants reveal conformational changes in $936 \mathrm{~L} 16$ loop that induce autophosphorylation and activation. J. Mol. Biol. 937 2007, 365, 66-76.

938 (70) Roskoski, R., Jr Src protein-tyrosine kinase structure, 939 mechanism, and small molecule inhibitors. Pharmacol. Res. 2015, 940 94, 9-25. 